\section{Dissecting out the role of the CCK-2 receptor in the carcinogenic effects of progastrin in the colon}

\section{Cerys Mayne, Anna Grabowska \& Sue Watson ${ }^{\dagger}$}

†Author for correspondence: Division of Pre-Clinical Oncology, School of Clinical Sciences, University of Nottingham, Nottingham, UK = Tel.: +44 1158231135 = Fax: +44 1158231137 = sue.watson@nottingham.ac.uk

Evaluation of: Jin G, Ramanathan V, Quante M et al:: Inactivating cholecystokinin-2 receptor inhibits progastrin-dependent colonic crypt fission, proliferation, and colorectal cancer in mice. J. Clin. Invest. 119 (9), 2691-2701 (2009). This paper investigates the roles of progastrin and CCK-2R in colon cancer using transgenic

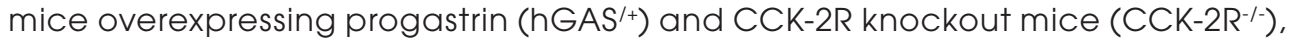
treated with the carcinogen azoxymethane. The data support a role for CCK-2R in mediating the proliferative effects of progastrin and the formation of aberrant crypt foci. However, the CCK-2R $/$ - mice showed elevated levels of amidated gastrin, which may mediate some of the effects observed, and direct signaling of progastrin through CCK-2R is not demonstrated. Increased expression of $\beta$-catenin and its downstream target, CD44, and of the putative stem/progenitor cell marker, DCAMKL1, in the hGAS ${ }^{/+}$mice, suggest a protective role for progastrin and CCK-2R on stem cells in the colon. These findings need further investigation in relation to elucidating a potential role for CCK-2R in promoting the survival of cancer stem cells.

\section{Summary of methods \& results}

Progastrin has an established role in colon carcinogenesis, but the receptor through which it mediates these effects remains elusive. The classical gastrin/CCK-2 receptor (CCK-2R) has been indicated to be overexpressed in colon cancer, but as it binds fully processed amidated gastrin, its role in progastrin-induced biological effects is controversial. This paper set out to investigate the combined roles of progastrin and the CCK-2R in colon cancer, by cross-breeding transgenic mice overexpressing the human form of progastrin, hGAS ${ }^{/+}$mice, with mice in which the murine CCK-2R was deleted (the CCK-2 $\mathrm{R}^{-1-}$ mouse) and characterized the resultant phenotype [1]. Initial findings confirmed, by in situ hybridization, that the CCK-2R was localized to the base of colonic crypts in wildtype (WT) mice and hGAS ${ }^{\prime+}$ mice, but in the latter was overexpressed and, as expected, not present in CCK-2R $\mathrm{R}^{-1-}$ mice. This was confirmed by real-time reverse-transcription (qRT)-PCR, and expression was increased at early time points (premalignancy) following treatment with the carcinogen, azoxymethane (AOM).

The crossed mice (hGAS ${ }^{/+} \mathrm{CCK}-2 \mathrm{R}^{-1-}$ ) were then used to investigate the role of CCK-2R in progastrin-induced proliferation of the colonic mucosa, and these results were compared with those of both of the original mouse models (hGAS ${ }^{/+}$and CCK-2R $\mathrm{R}^{-/}$), as well as the WT mice. Bromodeoxyuridine (BrdU) incorporation studies demonstrated that the cells at the base of the crypts were proliferating faster than those elsewhere, and that hGAS ${ }^{\prime+}$ mice demonstrated significantly higher BrdU-positive cells than the other three groups. To link this to the main objective of the study, it would have been interesting to confirm by immunofluorescent co-staining on cryopreserved sections that the proliferating cells were indeed expressing CCK-2R. Interestingly, the CCK-2R $\mathrm{R}^{-/}$and $\mathrm{hGAS}^{/+} \mathrm{CCK}-2 \mathrm{R}^{-1-}$ mice showed higher levels of apoptotic cells in the mucosa by terminal deoxynucleotidyl transferase dUTP nick-end labeling (TUNEL) assay. The authors suggest that this indicates that progastrin inhibits colonic mucosa apoptosis, but since the levels of TUNEL-positive cells in hGAS ${ }^{\prime+}$ mice are very similar to those in the WT, this does not seem to be the case. The lack of CCK-2R appears to be the real cause of the apoptosis, since both CCK-2R $\mathrm{R}^{-1-}$ groups have high levels of TUNELpositive cells. In addition, it would have been useful to further analyze which type of cells were apoptosing to determine if they were cells in the stem cell niche that were potentially responding to murine serum amidated gastrin.
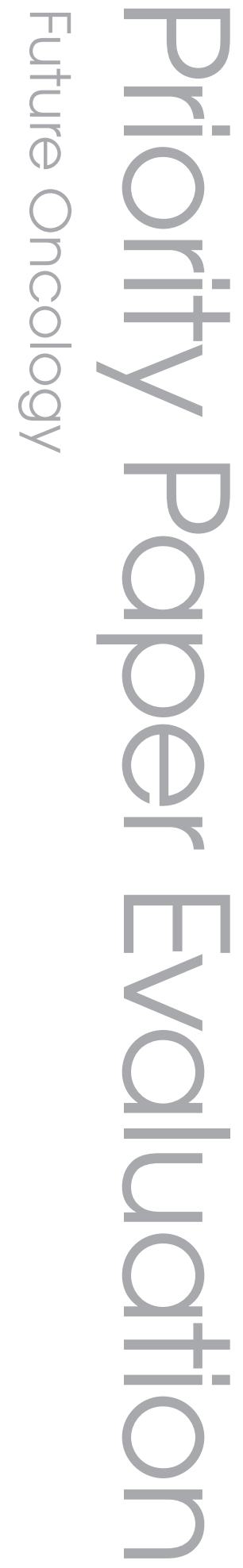

\footnotetext{
Keywords

= CCK-2R = colonic crypt

- colorectal cancer

- gastrin $=$ progastrin

- stem cell niche
}

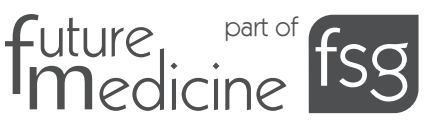


The upregulation of progastrin in $\mathrm{hGAS}^{/+}$ mice resulted in the formation of aberrant crypt foci (ACF) following exposure to AOM; however, this was significantly reduced in hGAS ${ }^{\prime+}$ CCK- $2 \mathrm{R}^{-/-}$mice when compared with hGAS ${ }^{/+}$ mice. As expected, the CCK- $2 \mathrm{R}^{-1-}$ mice had reduced $\mathrm{ACF}$, but interestingly this was also true in comparison with WT mice, although the significance of this is not shown.

Following longer AOM exposure, resultant tumor burden was significantly reduced in both CCK- $2 \mathrm{R}^{-/}$mouse strains when compared with $\mathrm{hGAS}^{/+}$mice, and when CCK- $2 \mathrm{R}^{-1-}$ mice are compared with WT mice, not only in number but also size. Consequently, in mortality studies the hGAS ${ }^{/+}$mice started to die at 17 weeks, compared with 27 weeks for the hGAS ${ }^{/+}$CCK- $2 \mathrm{R}^{-1}$ mice, with 0 and $70 \%$ of mice, respectively, surviving the 40 -week study. Interestingly, progastrin affected the distal colon to the greatest extent, and it would have been interesting to perform a proximal to distal comparison of expression of CCK-2R within the stem cell niche.

As expected, progastrin levels in both hGAS ${ }^{\prime+}$ variant mice were highly elevated compared with the WT and CCK- $2 \mathrm{R}^{-1-}$ groups. However, the amount of amidated gastrin present in the plasma was significantly higher in the hGAS ${ }^{/+}$ CCK- $2 \mathrm{R}^{-1}$ and CCK- $2 \mathrm{R}^{--}$groups, as previously reported [2]. This would indicate that the mice were hypo-acidic, and this rise in amidated serum gastrin was as a result of a feedback loop.

An early indicator of progression to colon cancer is the activation and nuclear localization of $\beta$-catenin, which has previously been linked to progastrin [3]. Expression of this molecule was found to be similar between all of the four groups of mice, before treatment with AOM, and increased in them all after treatment. However, the only mice that showed nuclear localization of $\beta$-catenin were the hGAS ${ }^{\prime+}$ group. CD 44 , a downstream target of $\beta$-catenin, was also found in all the groups, but the hGAS ${ }^{/+}$mice exhibited higher levels than the WT and especially the two CCK- $2 \mathrm{R}^{-1}$ groups. However, again there did appear to be differences between WT and CCK-2 $\mathrm{R}^{-/}$, suggesting that CCK-2R mice were responsive to murine amidated gastrin in a similar manner.

Another marker that has higher expression patterns in the $\mathrm{hGAS}^{/+}$as opposed to the $\mathrm{hGAS}^{/+}$ CCK- $2 \mathrm{R}^{-1-}$ and CCK-2 $\mathrm{R}^{-1-}$ mice was DCAMKL1, a putative stem/progenitor cell marker. This was shown to be present in the base of the colonic crypts in the hGAS ${ }^{/+}$mice, but further up the crypt walls, and less common, in the other three groups, indicating a possible effect of progastrin on crypt cell migration. It would be interesting to again perform co-staining with CCK-2R and repeat this finding using antibodies directed against Lgr5. Increased numbers of stem cells are thought to lead to increased crypt fission, which in turn result in increased risk of progression to colonic carcinoma [4]. Furthermore, the damaging effect of $\gamma$-radiation on these DCAMKL1-positive cells was decreased in the $\mathrm{hGAS}^{/+}$mice compared with the WT, implying a protective effect for progastrin on stem cells in the colon. Interestingly, when compared with WT mice, CCK- $2 \mathrm{R}^{-1}$ mice had similar levels of DCAMKL-1 positive cells, indicating that lack of CCK-2R does not affect stem cell number.

When CCK-2R was inhibited via YM022, a specific CCK-2R antagonist, the BrdU-positive proliferating cells in $\mathrm{hGAS}^{\prime+}$ mice were reduced significantly, supporting the results achieved with genetic deletion in the hGAS ${ }^{\prime+} \mathrm{CCK}-2 \mathrm{R}^{-1-}$ mice. In separate experiments, the inhibitor also caused decreased crypt fission, increased the number of TUNEL-positive apoptotic cells and reduced the formation of ACFs in the hGAS ${ }^{/+}$ group, all mimicking the outcome of the earlier assays in $\mathrm{hGAS}^{/+} \mathrm{CCK}-2 \mathrm{R}^{-/-}$mice. However, as is the case for the CCK- $2 \mathrm{R}^{-/-}$studies, inhibition of the progastrin-mediated effects does not demonstrate that the effects of progastrin are mediated directly through the CCK-2R. Instead, progastrin acting through another receptor may trigger events that lead indirectly to increased signaling through the CCK-2R.

\section{Discussion}

The initial case made for the role of CCK-2R in colon cancer progression has been fully supported by the results described above. The authors also put forward a convincing argument for the involvement of the CCK-2R in mediating the proliferative effects of progastrin. However, in general the discussion in this observational paper could have been strengthened by further focus on the stem cell link to progastrin.

Apoptosis, a process that is usually decreased in cancer progression, is seen to be upregulated to similar levels in both $\mathrm{hGAS}{ }^{/+} \mathrm{CCK}-2 \mathrm{R}^{-/}$and CCK-2 $\mathrm{R}^{-1-}$ mice, compared with the $\mathrm{hGAS}^{\prime+}$ and WT groups. This, along with the YM022 inhibitor study, implies that the lack of CCK-2R can significantly increase apoptosis, allowing cancer cells to be removed. The authors propose that progastrin inhibits apoptosis in the colonic mucosa, but they have not proved this since WT and hGAS ${ }^{++}$mice have similar numbers of 
apoptotic cells, yet the former lack progastrin. Similarly, whilst there are increased numbers of apoptotic cells in the CCK2 $\mathrm{R}^{-1}$ mice, there is not a significantly greater increase in the $\mathrm{hGAS}^{/+}$ group, and thus it is not necessarily the case that this is a result of progastrin signaling via the CCK-2R, but is more likely due to loss of signaling through the CCK-2R by endogenous gastrin, which is elevated in these two groups, indicating that CCK-2R may protect the colon stem cell niche.

The authors refer to a study by Cobb et al. that found that amidated gastrin may have a protective effect on the colonic mucosa, against the initiation and progression of carcinogenesis [5]. They found that gastrin knockout mice were more likely to develop ACFs and showed higher numbers of BrdU-positive cells than WT mice when treated with AOM. However, the two studies are difficult to compare, as the mice in the present study with elevated amidated gastrin have no CCK-2R to mediate any effects, and in $\mathrm{hGAS}^{+}$mice amidated gastrin is not completely eliminated. Thus, human progastrin is not present on a background of no murine gastrin, and the effects of progastrin on ACF and tumor formation may not therefore be partially explained by the lack of signaling mediated by murine amidated gastrin.

Another interesting discussion point was that the CCK-2R may be a progastrin gene target, and that this indirectly affects the ability of cancer stem cells to respond to amidated gastrin. This possibility needs to be further explored, and links to further investigation surrounding the regulation of gene and protein expression of the CCK-2R now that superior investigational tools are available.

\section{Future perspective}

Taken together, the results of this study suggest that progastrin, mediated by the CCK-2R, promotes colon cancer cell proliferation, inhibits apoptosis and protects and encourages stem cell expansion in the base of the colonic crypts. This in turn leads to increased crypt fission, and not only increased numbers, but also higher carcinogenicity, of ACFs and tumors. This role of the CCK-2R at the level of the intestinal stem cell needs much further investigation in parallel with other stem cell markers such as Lgr5, and to be expanded to investigate its role in cancer stem cells. The procarcinogenic activity of progastrin needs to be further probed to investigate its role in downstream gene expression, and it offers a new target for agents targeting either its production, its protein-protein interactions within the cytoplasm or its function (i.e., neutralizing antibodies).

\footnotetext{
Financial \& competing interests disclosure

The authors have no relevant affliations or financial involvement with any organization or entity with a financial interest in or financial conflict with the subject matter or materials discussed in the manuscript. This includes employment, consultancies, honoraria, stock ownership or options, expert testimony, grants or patents received or pending, or royalties.

No writing assistance was utilized in the production of this manuscript.
}

\section{Bibliography}

1. Jin GV, Ramanathan M, Quante GH et al: Inactivating cholecystokinin-2 receptor inhibits progastrin-dependent colonic crypt fission, proliferation, and colorectal cancer in mice. J. Clin. Invest. 119(9), 2691-2701 (2009).

2. Rindi G, Langhans N, Rehfeld JF, Beinborn M, Kopin AS: Abnormal gastric morphology and function in CCK-B/gastrin receptor-deficient mice. Yale J. Biol. Med. 71(3-4), 347-354 (1998).

3. Pannequin J, Delaunay N, Buchert $\mathrm{M}$ et al.: $[\beta]$-catenin/Tcf- 4 inhibition after progastrin targeting reduces growth and drives differentiation of intestinal tumors. Gastroenterology 133(5), 1554-1568 (2007).
4. He XC, Yin T, Grindley JC et al.: PTEN-deficient intestinal stem cells initiate intestinal polyposis. Nat. Genet. 39(2), 189-198 (2007).

5. Cobb S, Wood T, Tessarollo L et al.: Deletion of functional gastrin gene markedly increases colon carcinogenesis in response to azoxymethane in mice. Gastroenterology 123(2), 516-530 (2002).

\begin{tabular}{l}
\hline Affiliations \\
Cerys Mayne \\
Division of Pre-Clinical Oncology, School \\
of Clinical Sciences, \\
University of Nottingham, \\
Nottingham, UK
\end{tabular}

- Anna Grabowska Division of Pre-Clinical Oncology, School of Clinical Sciences, University of Nottingham, Nottingham, UK

- Sue Watson

Division of Pre-Clinical Oncology, School of Clinical Sciences, University of Nottingham, Nottingham, UK

Tel.: +441158231135

Fax: +441158231137

sue.watson@nottingham.ac.uk 